SHS Web of Conferences 20, 01003 (2015)

DOI: $10.1051 /$ shsconf $/ 20152001003$

(C) Owned by the authors, published by EDP Sciences, 2015

\title{
Construire une démarche à visée réflexive sur l'activité prescriptive des enseignants : choix méthodologiques
}

\section{Building a reflective approach to teachers' prescriptive activities: methodological choices}

\author{
Estefanía Domínguez ${ }^{1, a}$ \\ ${ }^{1}$ EA2268 DILTEC, Université Paris 3 - Sorbonne Nouvelle, 75005 Paris, France
}

\begin{abstract}
Résumé. Notre recherche porte sur le métier d'enseignant et plus particulièrement sur la passation de consignes en classe de français langue étrangère (FLE), pratique que nous abordons sous l'angle de la réflexivité. Nous traiterons dans cet article des choix méthodologiques de cette étude en explicitant le cheminement du chercheur lors de l'articulation des objectifs et des outils de recherche. Nous présenterons donc ces choix mais également les contraintes, difficultés et renoncements inhérents à ce processus. Après avoir contextualisé notre recherche, nous présenterons concrètement la démarche réflexive mise en place. Nous définirons les objectifs des quatre paliers qui structurent la démarche, à savoir questionnaire, réunion-échange, journal de bord et entretien d'autoconfrontation, tout en indiquant les modalités de réflexion ainsi que les types de discours produits par les enseignants. Il s'agira également de préciser le rôle, l'engagement et la relation entre les enseignants-participants et le chercheur afin d'évoquer l'influence de ces paramètres dans le déroulement de la recherche.
\end{abstract}

\begin{abstract}
Our research focuses on the act of teaching. Specifically, we will focus on giving instructions within the context of teaching French as a foreign language. We will approach the subject from a reflective perspective. We will identify the methodological choices for this study by exploring the researcher's point of view at the time the objectives and research tools were developed. We will present the researcher's choices as well as limitations, difficulties and renunciations inherent in such a process. After contextualising our research, we will present the reflective approach in concrete terms. We will define the objectives of the four stages that make up the process: that is the questionnaire, the meeting-exchange, the journal writing and the selfevaluation interview, explaining the method of reflexion as well as the types of discourse employed by teachers. We will also predict the roles, engagement and relationships between teachers, students and researchers in order to identify the influence said relationships have on the research process.
\end{abstract}

\section{Introduction}

À la suite de Donald Schön (1994 [1]), le paradigme du praticien réflexif imprègne les recherches et les programmes de formation ${ }^{\mathrm{b}}$. Penser la profession et la formation enseignantes implique de se pencher entre autres sur la question de la pratique réflexive, c'est-à-dire sur le « dialogue entre la pensée et l'action » (Legault, 2004 [3]) des enseignants. Entre autres, les questionnements sur les méthodologies, sur les stratégies d'enseignement-apprentissage, sur les modalités de travail, sur l'efficacité des pratiques

\footnotetext{
${ }^{a}$ Auteur de correspondance : estefania.dominguez.fle@gmail.com

${ }^{b}$ Les influences de Schön sont amplement développées par des chercheurs nords-américains et européens dans le récent ouvrage intitulé Le virage réflexif en éducation. Où en sommes-nous 30 ans après Schön ? (Tardif \& al., $2012[2])$.
} 
de cours sont partie intégrante du métier et ce, tout au long de la carrière. Cette réflexion peut être menée en autonomie ou être accompagnée. Les dispositifs qui encouragent ces pratiques réflexives chez les futurs enseignants sont de plus en plus nombreux, notamment dans les formations initiales (par exemple, par le biais de journaux de bord). Pour ce qui est des enseignants experts, les dispositifs sont moins nombreux, or il nous semble que la pratique réflexive permet une mise à distance du métier qui ne peut être que pertinente et enrichissante, quelle que soit l'expérience du sujet, car elle participe au développement professionnel. Par ailleurs, les recherches qui portent sur l'agir professoral et qui sollicitent pour cela les discours des enseignants (soit à travers des entretiens d'explicitation ou d'autoconfrontation) sont des clés d'accès aux savoirs pratiques de ce genre professionnel, ce qui permet aux enseignants et aux chercheurs de rentrer dans les coulisses du métier : accès aux représentations, aux stratégies mises en place, à la gestion des imprévus, aux conceptions des rôles des apprenants, aux difficultés rencontrées, aux émotions, au vécu, etc. ; en bref à une « véritable malle au trésor » (Cicurel, 2011 [4]). Dans le cadre de notre recherche doctorale en cours, nous sollicitons des discours écrits et oraux, d'enseignants de FLE expérimentés (plus de vingt ans d'expérience enseignante), axés sur la passation, transmission et gestion de consignes en classe. Nous nous proposons dans cet article d'expliciter le cheminement effectué lors des prises de décisions nécessaires à la définition de notre méthodologie de recherche. Pour ce faire, tout d'abord, nous poserons le socle théorique dans lequel nous nous inscrivons. Ensuite, nous présenterons succinctement nos objectifs de recherche, pour enfin, développer plus amplement l'articulation entre ces objectifs de recherche et les outils de recueil de données retenus.

\section{Vers les choix méthodologiques}

Pour aborder les choix méthodologiques effectués, il nous faut, dans un premier temps, contextualiser notre recherche. Nous présenterons donc quelques éléments théoriques centraux, notre positionnement par rapport aux rôles des enseignants et du chercheur, ainsi que l'origine de la recherche.

\subsection{Considérations théoriques}

L'interactionnisme socio-discursif (Bronckart, 1997 [5]) situe la parole au cœur des études sur l'agir humain. Dans les études récentes sur le métier d'enseignant (Bucheton, 2009 [6] ; Cicurel, 2011 [4] ; Jorro, 2002[7]) la parole est mise au premier plan par la sollicitation de verbalisations d'enseignants sur leur activité. Il est alors considéré que l'agir enseignant « peut être connu à la fois par l'observation des interactions et par les dires des acteurs à propos de l'action » (Cicurel, 2011 [4]).

Nous nous inscrivons alors dans les recherches qui portent sur l'agir professoral. Celui-ci est entendu comme «l'ensemble des actions verbales et non verbales, préconçues ou non, que le professeur met en place pour communiquer des savoirs à un public donné dans un certain contexte » (Cicurel, 2011 [4]). Notons dans cette définition la prise en compte du non-verbal, souvent considéré comme secondaire ou inné. Les éléments non-verbaux sont d'autant plus centraux car la communication chez les enseignants est composée de paroles, de gestes, de regards, de mouvements du corps, de pointages, de pauses, etc. Selon Jean-Marc Coletta, « dans la parole, la langue ne se présente jamais nue mais au contraire toujours habillée du costume de la voix du locuteur et du pardessus de ses attitudes, gestes, mimiques et regards » (Coletta, 2005 [8]). Par conséquent, nous ne pouvons dissocier le verbal du non-verbal, et pour le cas du métier qui nous occupe, ces composantes font partie des « outils » dont dispose l'enseignant.

Au sein de l'agir professoral, l'une des pratiques dont use l'enseignant est l'activité de prescription. Celle-ci a été amplement étudiée par Véronique Rivière qui la définit comme « l'activité langagière [...] qui incite à l'action et vise la transformation de l'autre et, en l'occurrence de savoirs et savoir-faire langagiers dans la langue étrangère ou seconde [et qui] se déploie en injonctions (dire de faire), en consignes liées à une tâche (dire quoi faire), ou en instructions (dire comment ou pourquoi faire) " (Rivière, 2008 [9]). La passation, transmission et gestion de consignes s'effectuent dans le vif de l'action de la classe où s'imbriquent, entre autres, paroles, gestes, regards et ressources matérielles et ce, selon les objectifs qui sous-tendent la consigne ou selon la réalité du contexte. Lors d'une étude précédente 
(Dominguez, 2013 [10]), nous avons constaté que des enseignants de FLE avaient une forte conscience des éléments verbaux en matière de passation de consignes et une moindre conscience des éléments non-verbaux. Dans le cadre de notre recherche doctorale, au regard du paradigme du « praticien réflexif » (Schön, 1994 [1]), nous avons émis l'hypothèse qu'une étude plus approfondie, étayée par des outils favorisant le développement de pratiques réflexives, pourrait amener d'une part à une compréhension plus fine de l'activité de prescription et d'autre part à une meilleure conscientisation de la multimodalité de l'agir prescriptif par les enseignants.

Dans cette recherche, l'un des buts est donc d'engager les enseignants dans une démarche de réflexion et de réflexivité. Cette dernière consiste à « réfléchir sur l'action [et] prendre sa propre action comme objet de réflexion » (Perrenoud, 2010 [11]). L'activité réflexive peut avoir lieu : avant, pendant ou après l'action, ce qui suppose pour le professionnel de prendre du recul et d'analyser son propre fonctionnement (Altet \& al., 2012 [12]). Cependant, l'activité réflexive ne va pas de soi, par exemple, dans le vif de l'action, elle est partiellement inaccessible à notre conscience ; par conséquent « elle soulève [...] des résistances [mais] des dispositifs peuvent la favoriser » (Altet \& al., 2012 [12]). Nous verrons plus loin les dispositifs que nous avons choisis afin de favoriser l'activité réflexive des enseignants-participants.

\subsection{De la définition des caractéristiques de la démarche réflexive : positionnements de départ}

Comme nous l'avons signalé, nous avons comme objectif de mener une recherche à caractère réflexif chez des enseignants experts de FLE sur leur activité de prescription. De façon générale, deux questions de recherche sous-tendent cette optique : dans quelle mesure une démarche réflexive pourrait-elle aider à un approfondissement de la connaissance de l'agir prescriptif ?, en quoi une démarche réflexive amènerait-elle une plus forte verbalisation de la dimension multimodale de la transmission-gestion de consignes? Afin de répondre à ces questions, il nous fallait donc réaliser des choix pour construire une démarche réflexive, ce qui suppose avant tout de définir les caractéristiques dont elle devait disposer.

Dans un premier temps, nous nous sommes penchée sur la question des enjeux liés à la forte participation des enseignants à notre recherche. D'une part, d'un point de vue pragmatique et téléologique, nous devions mettre en place une démarche réflexive qui favorise la production de discours (écrits et oraux) sur l'agir prescriptif des enseignants sollicités. D'autre part, ces discours avaient pour objectif de permettre aux enseignants d'acquérir un bagage réflexif et linguistique sur la passation, la transmission et la gestion de consignes. Conséquemment, notre action allait demander un fort engagement des personnes participantes. Celui-ci passe, entre autres, par un facteur temporel non négligeable, à savoir une participation sur la durée : les enseignants, comme nous le verrons, sont amenés à répondre à des questionnaires et à participer à des échanges pendant plusieurs mois. Précisons dès à présent, que lors de la prise de contact, tous ont été informés de cette « contrainte ». Les personnes qui participent à cette recherche sont donc conscientes en amont de ce qui leur sera demandé. Cette transparence était pour nous une condition sine qua non, étant donné que sans un contrat de communication et de confiance instauré dès le début de la recherche, les garanties de participation et d'implication seraient moindres. Comme le signale Mireille Cifali, « il n'y a pas d'action sans un sujet qui la porte. Pas de pensée de l'action sans un sujet qui s'y engage » (Cifali, 2008 [13]). Nous avons donc insisté sur la collaboration que cette recherche supposait; tous ont verbalisé leur désir et leur volonté de réfléchir sur leur métier et leur pratique. Nous pouvons supposer que cette acceptation signale « une volonté de remettre en question autant [leur] façon de penser que [leur] façon de faire » (Legault, 2004 [3]). Notons que les quatre étapes qui constituent notre démarche réflexive amènent notamment à une réappropriation du vécu des enseignants, ce qui peut donc avoir un impact sur la relation qu'ils entretiennent avec leur agir professoral et par là-même sur leur identité enseignante, leur " moi » professionnel. Comme le signale Yann Vacher « la sécurisation de l'engagement dans la pratique réflexive se développe à travers la reconnaissance et la prise en compte des angoisses [...] du praticien (Vacher, 2015 [14]). 
Par ailleurs, nous considérons les enseignants non seulement comme des informateurs mais également comme des « acteurs [et] véritables producteurs de savoirs » (Blanchet \& Chardenet, 2011 [15]). En effet, nous avons accès, entres autres, à leurs « savoirs pratiques » (Altet \& al., 2012 [12]) élaborés à partir de leur propre expérience. Ces savoirs émergeront tantôt dans des discours écrits tantôt dans discours oraux (qui constitueront un corpus de données multimodales, associant selon le cas, texte, son et image).

À cette étape, il est également nécessaire de traiter de la position du chercheur. Pour ce faire, nous nous appuyons sur les propos de Caroline Scheepers qui met en avant les caractéristiques de ce qu'est une " recherche impliquée ». Selon elle, " la posture du chercheur impliqué [...] se fonde [...] sur plusieurs courants de recherche qui contribuent à lui conférer sa légitimité : ethnométhodologie, ethnographie réflexive, pragmatisme, École de Chicago, interactionnisme symbolique » (Sheepers, 2010 [16]). Dans notre recherche nous sollicitons, nous accompagnons, nous recueillons et nous interprétons des discours réflexifs de natures diverses. En outre, nous partageons avec les participants la même profession (non pas la même expérience). Il s'agit donc d'une recherche qui peut être entendue comme impliquée et donc subjective. Pour une objectivisation et une analyse de la posture de recherche, Scheepers signale qu'elle est « revenue [dans le cadre de sa recherche] assez longuement sur la démarche par laquelle [sa] problématique définitive s'est établie, sur le processus par lequel [elle a mis] au point puis expérimenté [sa] constellation d'indicateurs de la réflexivité ou encore sur les principales orientations méthodologiques » (Sheepers, 2010 [16]), propos que nous partageons.

Notre recherche est une recherche qualitative qui s'étale dans le temps, qui suppose donc des prises de décisions et des réajustements liés au fait même de travailler sur des pratiques enseignantes par le biais d'une collaboration avec les acteurs de celles-ci, les enseignants-experts. De ce travail, sur et avec ces enseignants, découle une relation entre chercheur et informateurs qui ne peut être ignorée. En outre, notre rôle de chercheuse passe de « provocatrice » de discours réflexifs à " collaboratrice » dans le retour sur l'expérience, la pratique et les savoirs. Notre statut est alors partagé et se manifeste sous plusieurs casquettes : enseignante de FLE, chercheuse et formatrice. Ce sont alors l'inscription dans des cadres de références théoriques, les méthodes de recherches choisies et les techniques d'analyse qui viennent appuyer une certaine mise à distance de notre objet et de nos sujets-participants.

\subsection{Genèse de la recherche et impact sur les choix méthodologiques}

Avant de présenter chaque palier de la démarche réflexive ainsi que les choix et difficultés associés, nous allons contextualiser notre recherche doctorale, car ce sont la méthodologie et les résultats de notre mémoire de recherche de Master qui, dans un premier temps, ont influencé les choix méthodologiques de la présente recherche.

Comme nous l'avons signalé précédemment, nous avons réalisé en 2013 (Dominguez, 2013 [10]) une étude sur le degré de conscientisation de la multimodalité de l'activité de prescription. Lors de cette recherche, nous avons filmé des enseignants de FLE et réalisé des entretiens d'autoconfrontation lors desquels nous convoquions une mise en mots sur ladite activité. L'étude elle-même, ainsi que nos lectures, nous ont amenée à des considérations et positionnements divers.

Dominique Bucheton considère que « le métier d'enseignant est peu "parlé » par les enseignants eux-mêmes, sans doute parce que difficile à dire, sans doute aussi parce que le huis clos de la classe n'ouvre que timidement sa porte » (Bucheton, 2011 [17]). A cet égard, l'auteure met en avant le rôle de la verbalisation en entretien d'autoconfrontation car celle-ci se révèle être un moyen efficace pour « nommer [et] expliquer [les] pratiques » (Bucheton, 2011 [17]).

Notre positionnement repose donc sur l'affirmation suivante : pour que des enseignants de FLE puissent réaliser un travail de réflexion plus approfondi sur l'activité de prescription (et en deçà, sur l'aspect multimodal de celle-ci) et pour que ce processus réflexif permette éventuellement une transformation de leur pratique, nous considérons qu'il pourrait être pertinent de les engager dans une activité réflexive « durable ». C'est pour cela que, de par la revue de littérature que nous avons faite sur les outils réflexifs qui existent, nous avons combiné quatre outils : le questionnaire écrit, un échange de pratiques, un journal de bord et un entretien d'autoconfrontation. En structurant de la sorte nos outils de 
recueil de données, nous engagerions donc les enseignants dans une dynamique réflexive d'abord individuelle (le questionnaire), ensuite collective (l'échange), puis une nouvelle fois individuelle (le journal de bord) et enfin en collaboration avec le chercheur (l'entretien d'autoconfrontation). Ces quatre temps de réflexion permettraient donc d'enrôler progressivement les enseignants dans une forte activité réflexive qui, par les différentes occasions de mise en mots (écrites ou orales, individuelles ou collectives) pourraient a priori leur permettre d'être plus outillés au moment de l'entretien d'autoconfrontation.

\section{L'articulation entre les objectifs et les outils de recherche}

Comme indiqué, nous avons construit un dispositif de recherche qui consiste en une démarche réflexive. La démarche doit être entendue comme une suite de paliers, chacun répondant à des objectifs précis et dans une suite logique :

- premier palier : un questionnaire

- deuxième palier : une réunion-échange

- troisième palier : un journal de bord

- quatrième palier : un entretien d'autoconfrontation

Comme nous allons le voir ci-après, chaque palier amène un type et une modalité de réflexion précis qui tournent autour du pivot central : l'activité de prescription.

\subsection{Le questionnaire : objectifs, choix et difficultés}

Tableau 1. Descriptif du questionnaire

\begin{tabular}{|l|l|l|}
\hline Description de l'outil & Objectifs de l'outil & Travail réflexif chez l'enseignant \\
\hline $\begin{array}{l}4 \text { thèmes : l'enseignement, } \\
\text { l'apprentissage, les consignes } \\
\text { et la pratique réflexive }\end{array}$ & $\begin{array}{l}\text { - Faire émerger les représentations } \\
\text { - Engager les participants dans un } \\
\text { processus réflexif sur le métier enseignant } \\
\text { et sur la passation de consignes }\end{array}$ & Réflexion individuelle à l'écrit \\
\hline
\end{tabular}

Le choix du premier palier relève de l'objectif de poser un premier espace de réflexion général. Par le biais de ce questionnaire, il s'agit d'avoir accès aux « représentations, croyances, savoirs » (CambraGiné, 2003 [18]) et aux pratiques déclarées ${ }^{\mathrm{c}}$ des enseignants-participants. Par ailleurs, cette étape favorise « un processus individuel de subjectivation auto-adressée qui pousse le scripteur à prendre du recul par rapport à son objet et à s'extraire de son inscription dans l'immédiat [ce qui] crée chez le scripteur [...] une sorte de dialogue avec lui-même, un rapport à une altérité interne » (Bibauw \& Dufays, $2010[19])^{\mathrm{d}}$.

En ce qui concerne l'étendue du questionnaire, nous avons décidé de ne pas faire l'économie des questions, non seulement parce que chacune répond à un objectif précis, mais aussi pour que ce palier permette une immersion dans le sujet de recherche et par là-même une plus forte activité de réflexion. Nous désirons en effet faire émerger des représentations et ressentis sur, entre autres, les enjeux liés aux consignes (formulation, difficultés de transmission et de gestion, la part du non-verbal dans la transmission, etc). Afin de permettre aussi des ajouts de leur part et d'encourager la réflexion, nous avons décidé de proposer un espace de réflexion libre après les deux premiers thèmes (cf. tableau 1, cidessus) où les enseignants peuvent ajouter des éléments, préciser des points ou proposer des sujets qui n'ont pas été abordés. En fin de questionnaire, dans cette même logique d'espace d'expression, nous

\footnotetext{
c Pour le thème des consignes, des questions proposées sont par exemple « quels sont les problèmes que posent les consignes ?, pour vous, pour les apprenants », « quels sont les critères qui motivent une reformulation de consignes ? ».

${ }^{\mathrm{d}}$ Bien que la production finale (le questionnaire complété) soit alter-adressée (le lecteur est bien le chercheur), ce mouvement de subjectivation auto-adressé est manifeste. Pensons aux questionnaires de recherche que nous recevons et auxquels nous participons, il y toujours une part de « mise à distance » souvent liée à une prise de conscience.
} 
proposons aux enseignants de mettre en mots les éventuels apports du questionnaire en tant que première étape réflexive.

Signalons que ce questionnaire facilite également l'émergence d'éléments « biographiques 》 des enseignants-participants (vécus, expériences diverses, principes), ce qui permet dans une certaine mesure d'avoir accès à une partie de leur « répertoire didactique » ${ }^{\mathrm{e}}$ (Cicurel, 2002 [20]; Cadet \& Causa, 2005 [21]). Ces éléments pourront donc permettre de préciser ou de contextualiser notre travail d'analyse de données.

Pour ce qui est des difficultés liées à ce premier palier, la longueur du questionnaire aurait pu donner lieu à des réponses succinctes ou encore à un faible taux de participation dans les parties consacrées aux ajouts (suite aux deux premiers thèmes) et à la partie finale de bilan. Or nous pouvons dire que les enseignants ont rempli leur contrat bien au-delà de nos attentes. Aussi, notre estimation erronée du temps nécessaire pour répondre au questionnaire aurait pu être un frein dans l'avancement de notre recherche car l'engagement temporel est conséquent tout comme l'engagement émotionnel ${ }^{\mathrm{f}}$. Ce point aurait donc pu nuire à la relation entre les enseignants et le chercheur.

\subsection{La réunion-échange : objectifs, choix et difficultés}

Tableau 2. Description de la réunion-échange

\begin{tabular}{|l|l|l|} 
Description de l'outil & Objectifs & $\begin{array}{l}\text { Travail réflexif chez } \\
\text { l'enseignant }\end{array}$ \\
\hline $\begin{array}{l}\text { Partie 1) paramètres liés à la } \\
\text { consigne (produits par les } \\
\text { participants) }\end{array}$ & $\begin{array}{l}\text { - Favoriser l'échange entre pairs en leur offrant un } \\
\text { espace de communication et de co-élaboration } \\
\text { Partie 2) présentation de cas } \\
\begin{array}{l}\text { Partie 3) retour et apports (par } \\
\text { le chercheur) }\end{array}\end{array}$ & $\begin{array}{l}\text { problémation en mots une situation concrète et à la passation de consignes } \\
\text { - Faire émerger les conceptions et pratiques d'une } \\
\text { communauté professionnelle }\end{array}$ \\
\hline
\end{tabular}

Comme nous l'avons signalé, la démarche réflexive que nous avons mise en place se propose de permettre, non seulement des espaces de réflexion individuels mais aussi collectifs. Parmi les objectifs de ce deuxième palier (cf. tableau 2, ci-dessus), il s'agissait d'accéder au « genre professionnel » (Clot \& Faïta, 2000 [23]) qui est entendu comme « ce que les travailleurs d'un milieu donné connaissent ou voient, attendent ou reconnaissent, apprécient ou redoutent » (Clot \& Faïta, 2000 [23]).

Dans un premier temps, le chercheur, dans son rôle de provocateur de discours réflexifs sur l'objet, demande aux enseignants-participants d'échanger entre eux sur les paramètres liés aux consignes. Cette activité est féconde en discours sur leur pratique et sur leurs représentations concernant la consigne. Les enseignants ont spontanément décidé de réaliser une carte conceptuelle de tous les paramètres liés aux consignes, ce qui nous a permis d'avoir une trace de leurs savoirs mais aussi de leurs savoirs pratiques déclarés et ce, au travers des échanges et étapes de négociation (précisons que la réunion est également filmée). À ce stade, le chercheur est auditeur passif (Gadet, 2003 [24]). Pour clore l'échange entre les participants, le chercheur relève et synthétise ce qui est dit et complète les éléments liés aux consignes qui n'ont pas été abordés (à savoir, le rôle des gestes dans la passation de consignes). C'est à cette étape

\footnotetext{
e «Le « répertoire didactique » de l'enseignant, défini comme un ensemble hétéroclite de modèles, de savoirs, des situations sur lesquels un enseignant s'appuie. Ce répertoire se constitue au fil des rencontres avec divers modèles didactiques [...], par la formation académique et pédagogique [...] par l'expérience d'enseignement qui elle-même modifie le répertoire » (Cicurel, 2002 [20]).

${ }^{\mathrm{f}}$ Dans le domaine de l'analyse du travail et plus concrètement pour ce qui est de l'engagement du salarié dans l'entreprise, «l'engagement affectif se définit [...] comme le fait pour une personne de s'identifier aux valeurs de son organisation, de faire des efforts importants dans son travail pour contribuer à sa réussite et enfin de vouloir en rester membre » (Paillé, 2008 [22]). Notre recherche engage les enseignants dans le sens où un contrat s'établit et des attentes sous-tendent les relations entre participants et chercheur. C'est dans ce sens que nous entendons l'engagement émotionnel.
} 
que le chercheur se pose et assume son rôle de guide; il sort donc de son rôle d'auditeur passif ou d'observateur non-participant à celui d'acteur dans la co-construction des savoirs car il apporte des compléments théoriques tout en s'appuyant sur les savoirs initialement produits par le groupe.

Dans un deuxième temps, les enseignants ont été invités à échanger sur une expérience concrète. À tour de rôle, chaque participant a identifié une expérience vécue et interpellante sur une activité de prescription lui ayant posé souci afin de la présenter ensuite au groupe. Après avoir formulé le problème, les pairs ont réagi et commenté la situation partagée en ayant pour consigne de ne pas évaluer ou juger la situation. Cette étape s'inspirant des analyses de pratiques permet « l'échange avec autrui dans un contexte non normatif [qui favorise] la décentration de son propre point de vue et l'ouverture à des interprétations et pistes alternatives » (Beckerts \& François, 2013 [25]).

Les difficultés associées à ce palier tiennent aux choix effectués en amont de sa réalisation. En effet, nous avions décidé, lors de la conception du dispositif, que cette deuxième étape de la démarche serait une occasion d'outillage théorique et conceptuel apporté par le chercheur. Suivant les préceptes de Bucheton qui prône une « réflexivité instrumentée par des concepts théoriques » (Bucheton, 2011 [17]), nous considérions que cet outillage pouvait aider à la mise en discours des paliers 3 et 4 davantage axés sur la pratique. Cependant, nous avons renoncé à cet outillage pour plusieurs raisons. Tout d'abord, cela nous semblait trop directif, ensuite nous nous sommes interrogée sur notre légitimité en tant que jeune chercheuse, enfin il nous a semblé plus pertinent de partir de leurs propres conceptions car nous serions plus cohérente avec notre choix de placer les enseignants au cœur de la recherche. Par ailleurs, en les amenant à échanger sur la consigne et à partager une expérience concrète, nous avions en partie accès à certaines pratiques déclarées.

Ce palier joue également un rôle dans l'avancement de la démarche. Il permet d'un côté de favoriser la mise en mots autour de la consigne mais aussi de permettre la réalisation du palier 3 (cf. tableau 3 , ci-dessous). En effet, en fin de réunion, le chercheur explique en détail comment est envisagée la tenue des journaux de bord. Le jour même de la réunion, les enseignants reçoivent donc par courriel : la synthèse des échanges (paramètres liés aux consignes) ainsi que le journal de bord qu'ils doivent remplir.

\subsection{Le journal de bord : objectifs, choix et difficultés}

Tableau 3 : Description du journal de bord

\begin{tabular}{|l|l|l|}
\hline Description de l'outil & Objectifs de l'outil & $\begin{array}{l}\text { Travail réflexif chez } \\
\text { l'enseignant }\end{array}$ \\
\hline $\begin{array}{l}\text { Journal divisé en deux parties : } \\
\text { - Partie descriptive : sur le contexte (objectifs, type } \\
\text { d'activités, modalités, etc.) et la passation- } \\
\text { transmission-réception de consignes } \\
\text { - Partie réflexive : sur les ressentis, les émotions, } \\
\text { interrogations, etc. vécus lors du cours qui vient } \\
\text { d'être imparti }\end{array}$ & $\begin{array}{l}\text { - Accéder aux savoirs procéduraux } \\
\text { - Evaluer l'impact du palier } 2\end{array}$ & $\begin{array}{l}\text { Réflexion individuelle } \\
\text { «à chaud à l'écrit } \\
\text { Centration sur soi et } \\
\text { son activité effective }\end{array}$ \\
\hline
\end{tabular}

En ce qui concerne les choix liés à la modalité de la tenue du journal, il a été demandé aux enseignants de le remplir pendant deux semaines. Il y a là, la même volonté qui caractérise toute la démarche réflexive : l'enrôlement réflexif. Pour remplir cette tâche, les participants disposaient donc de leurs savoirs, compétences et expérience mais aussi de la synthèse du palier 2 qui leur avait été préalablement envoyée. Rappelons que cette synthèse doit être comprise comme un soutien à la tenue des journaux qui permette donc de réutiliser des éléments énoncés par les enseignants ou apportés par le chercheur. Par exemple, pour le cas des éléments non-verbaux (non abordés lors du premier temps de la réunionéchange), les discours écrits des journaux pouvaient montrer des parties descriptives ou réflexives sur les gestes employés. C'est dans ce sens que parmi nos objectifs, nous avions aussi l'évaluation de l'impact du palier 2.

Quant aux difficultés, notons tout d'abord qu'il s'agit d'une activité basée sur la confiance : le chercheur ne peut pas vérifier si l'écriture a lieu effectivement après le cours. Pour être au plus près de 
la réalité, nous avons établi que si la réflexion n'avait pas eu lieu à chaud ${ }^{\mathrm{g}}$, cela devait être signalé sur le journal (ce qui a d'ailleurs été fait par les enseignants). Précisons ici qu'il est nécessaire dans ce type de recherche engageante pour les informateurs et pour le chercheur, de nourrir une communication fréquente, claire et sincère qui est, à notre avis, un gage de réussite dans l'accomplissement des rôles de chacun et par là-même des données qui seront produites. Cet aspect qui peut sembler banale est pourtant capital et fait partie de notre « recherche engagée ».

Pour ce qui est d'une autre difficulté, précisons une « erreur » dans la tenue du journal par l'un des enseignants qui n'a pas complété la partie réflexive. Cela a été évoqué par l'enseignant en entretien d'autoconfrontation (palier 4, cf. tableau 4 ci-dessous). Il explique que cela est dû à la ressemblance de cette tâche avec celle qu'il doit accomplir dans son établissement. Etant donné qu'il avait pour habitude de réaliser une production essentiellement axée sur la description, la partie réflexive a donc été reléguée à un deuxième plan dans son cas ; c'est un des écarts avec lequel le chercheur doit composer.

\subsection{L'entretien d'autoconfrontation : objectifs, choix et difficultés}

Tableau 4. Descriptif de l'entretien d'autoconfrontation (EAC)

\begin{tabular}{|l|l|l|}
\hline Description de l'outil & Objectifs de l'outil & $\begin{array}{l}\text { Travail réflexif chez } \\
\text { l'enseignant }\end{array}$ \\
\hline $\begin{array}{l}\text { 1) Verbalisations (à partir des } \\
\text { cours vidéoscopés) }\end{array}$ & $\begin{array}{l}\text { - Permettre la mise en mots sur l'activité } \\
\text { effective } \\
\text { 2) Echanges sur les verbalisations } \\
\text { - Recenser les catégories avec lesquelles } \\
\begin{array}{l}\text { 3) Bilan de la recherche (sur } \\
\text { chaque palier et sur l'ensemble de } \\
\text { la démarche réflexive) }\end{array}\end{array}$ & $\begin{array}{l}\text { l'enseignant est capable de penser sa } \\
\text { propre activité } \\
\text { - Avoir accès aux apports de la démarchen individuelle à partir } \\
\text { des extraits audiovisuels } \\
\text { 2) Réflexion guidée à partir des } \\
\text { interprétations fournies par le } \\
\text { chercheur }\end{array}$ \\
\hline
\end{tabular}

Suite à l'activité individuelle des journaux de bord, nous avons filmé les enseignants-participants dans leur salle de cours. Ces supports audiovisuels ont été découpés afin de présenter aux enseignants les parties qui portaient sur leur activité de prescription. Nous avons donc, pour ce palier 4, effectué des entretiens d'autoconfrontation. Par la vidéo, ce type d'entretien permet la mise en miroir de l'interviewé, contexte qui l'aide à se replonger dans son vécu. D'après Cicurel, il y a un changement de rôle chez l'enseignant qui « passe d'acteur sur le terrain, tenant un rôle prédeterminé auprès de ses élèves [à] observateur de lui-même [car] sollicité pour produire de la signification à propos de ses actions [et il devient alors] chercheur dans le contexte » (Cicurel, 2004 [4]) ; c'est ce que font les enseignants, à différents degrés dans tous les paliers mais très concrètement lors de cette dernière étape.

Ce quatrième palier est considéré comme révélateur ou vérificateur de la démarche réflexive ellemême. En effet, c'est à ce stade que nous pourrons observer des indices de (trans)formation car, les enseignants-participants sont passés par différents stades et modalités de réflexion. En ce sens, ils pourraient être plus à même de mettre en mots leur pratique prescriptive (éléments verbaux et nonverbaux).

À la différence de notre première recherche, où les données recueillies étaient interprétées par le chercheur, nous avons ici décidé d'associer le point de vue des acteurs à l'analyse de l'activité. Ainsi, suite à une analyse croisée des données du questionnaire, des journaux de bord et des vidéos, nous avons émis des interprétations que nous avons soumises aux enseignants lors de l'entretien. Celles-ci portaient sur des éléments divers et variés, fortement liés à la singularité de chaque enseignant. À titre d'exemple, nous avons remarqué chez un enseignant, que le rôle de sa voix dans la passation de consignes et certaines difficultés associées à cela étaient manifestes dans le questionnaire, ainsi qu'observables dans la vidéo. Le participant a réagi ensuite en validant, infirmant ou nuançant ces interprétations. Cette analyse soumise par le chercheur permet de dépasser ses interprétations au moment de l'analyse des données car il y associe la verbalisation de l'acteur. Cela permet également de renforcer la place des

${ }^{\mathrm{g}}$ Lors de l'analyse de données, qui n'a pas encore été réalisée à ce jour, nous devrons tenir compte de cette variable, pourrait-il y avoir des différences entre des écrits plus ou moins proche du moment de l'action? 
enseignants dans le dispositif car ils sont, rappelons-le, au cœur de celui-ci et leur parole est le matériel dont dispose le chercheur afin d'avoir accès, entre autres, aux verbalisations sur leur action, aux raisons de leur agir et à leurs prises de décisions.

La dernière étape de ce palier consiste en un retour sur chacun des paliers. La parole est donnée aux enseignants pour qu'ils puissent exprimer leurs vécus, les apports, les difficultés, les retombées de leur participation à la recherche (sur eux, sur leur pratique et sur leur activité prescriptive) ainsi que les critiques du dispositif auquel ils ont participé pendant quelques mois. C'est l'occasion également pour le chercheur d'avoir accès aux effets de la démarche réflexive. Cependant, il ne faut pas considérer ces informations comme une vérité absolue ; la parole des enseignants est adressée au chercheur et se produit en face à face. À ce sujet, le contrat de communication et les liens tissés tout au long de la collaboration à la recherche sont des éléments clés bénéfiques pour cette étape.

Les enseignants sont très exposés lors de ce palier car nous nous appuyons sur leur image (corps, voix, pratique, interactions) et ils doivent mettre en mots leur action. Ces discours portent sur leur « moi » professionnel mais à cette étape c'est également le « moi » singulier qui se dévoile. Cette entrée dans l'intimité des enseignants ne peut s'envisager, d'un point de vue éthique, sans une relation qui tend vers la confiance et qui se base sur l'honnêteté des interactants.

En ce qui concerne les difficultés relatives à ce palier, citons d'abord les prises de décisions liées à la structure même de l'entretien. Une fois que nous avions fait le choix de soumettre des interprétations aux enseignants, il s'est avéré qu'elles étaient nombreuses et que toutes ne pouvaient être soumises en entretien, nous n'en avons alors retenu que certaines. La raison étant pragmatique car nous considérons que l'énergie et l'effort demandés aux enseignants lors de l'autoconfrontation sont déjà importants. Il nous a fallu aussi réaliser des ajustements in vivo, car certaines des interprétations que nous allions leur soumettre trouvaient déjà réponse ou validation au moment de l'autoconfrontation. Une autre difficulté est associée à l'exercice même de l'autoconfrontation et plus particulièrement à l'impact de l'image. Ainsi, un des enseignants, durant les premières minutes, était submergé par l'émotion car l'exercice de vidéo-confrontation lui renvoyait une image qui ne correspondait pas à ce qu'il pensait faire : ses déplacements et sa gestuelle l'ont ainsi fortement marqué. Cet événement montre que lorsque les enseignants sont dans le vif de l'action, il y a une partie de leur action sur laquelle ils n'ont pas prise.

\section{Conclusion}

Nous avons tenté dans cette contribution de montrer les décisions prises par le chercheur au moment de la conception de la démarche réflexive. Nous avons également exposé certaines difficultés liées à l'application des outils.

Nous avons vu que les quatre paliers décrits permettent, entres autres, d'engager les enseignantsparticipants dans une dynamique réflexive et d'avoir accès à différentes traces de leur réflexion. Les discours produits (données textuelles, audiovisuelles et sonores) nous permettent donc de disposer de corpus multimodaux.

Les choix opérés par le chercheur portent principalement sur l'adéquation des outils avec les objectifs généraux de la recherche. Nous avons expliqué l'organisation chronologique des outils en montrant les modalités de réflexion qu'ils engendraient. Par ailleurs, nous avons vu qu'une rétroalimentation entre les paliers était nécessaire afin de répondre aux objectifs généraux de notre étude. En effet, chaque palier, à plus ou moins fort degré, permet une articulation entre expérience et réflexivité. Cet aménagement, favorisé par la mise en mots de vécus singuliers réalisée individuellement ou collectivement, permet l'accès aux savoirs professionnels. L'activité réflexive amène l'enseignantparticipant à différentes prises de conscience qui peuvent le former ou le transformer grâce à l'émergence des différents niveaux de conceptualisation qu'il réalise. C'est donc au travers des activités langagières des enseignants que s'opère la compréhension de l'activité de passation de consignes pour les enseignants eux-mêmes et pour la recherche.

Enfin, ce parcours dans les coulisses d'une recherche qualitative de nature ethnographique, montre la place centrale des enseignants et du chercheur, au cœur du dispositif et qui, d'une certaine façon, « coconstruisent » les données. Comme le signale Francine Cicurel «c'est le praticien placé devant son 
action, mis sur le chemin de la réflexivité, qui initie ce travail de conversion de l'expérience en conceptualisation que doit poursuivre le chercheur. Ainsi les concepts en didactique s'originent dans l'expérience et viennent ensuite, en retour, la nourrir et l'enrichir en fournissant aux professeurs des « mots pour le dire » et au chercheur, des concepts pour aller plus loin » (Cicurel, 2015 [26]).

Les savoirs d'expérience et savoirs pratiques mis en lumière par cette recherche pourront être mis au profit de la formation initiale à travers, entre autres, l'exploitation des discours écrits, oraux et audiovisuels, préalablement sélectionnés, en tant que supports de formation. L'objectif étant de réduire la distance et de faire dialoguer les produits scientifiques des recherches et la formation initiale.

\section{Bibliographie}

1. D. Schön, Le praticien réflexif. À la recherche du savoir caché dans l'agir professionnel (Ed. Logiques, Montréal, 1994)

2. M. Tardif, C. Borges, A. Malo, (dir.) Le virage réflexif en éducation (De Boeck, Bruxelles, 2012)

3. J.P. Legault, Former des enseignants réflexifs (Logiques, Outremont, 2004)

4. F. Cicurel, Les interactions dans l'enseignement des langues. Agir professoral et pratiques de classe (Didier, Paris, 2011)

5. J.P. Bronckart, Activité langagière, textes et discours. Pour un interactionisme socio-discursif (Delachaux et Niestlé, Lausanne, 1997)

6. D. Bucheton (dir.), L'agir enseignant : des gestes professionnels ajustés (Octares, Toulouse, 2009)

7. A. Jorro, Professionnaliser le métier d'enseignant (ESF, Paris, 2002)

8. J.M. Coletta, FDM-R\&A, $\mathbf{n}^{\circ}$ spécial, 32 (2005)

9. V. Rivière, FDM-R\&A, 44, 51 (2008)

10. E. Dominguez, L'enseignant de FLE et l'activité de prescription : multimodalité (pré)réfléchie? Paroles sur l'agir professoral (2013) URL http://dune.univangers.fr/fichiers/20097748/20132MDLA1555/fichier/1555F.pdf

11. Ph. Perrenoud, Développer la pratique réflexive dans le métier d'enseignant (ESF, Paris, 2010)

12. M. Altet, J. Desjardins, R. Etienne, L. Paquay, Ph. Perrenoud, Former des enseignants professionnels. Quelles stratégies? Quelles compétences? (De Boeck, Bruxelles, 2012)

13. M. Cifali in : M.Cifali, F. Giust-Desprairies (dir), Formation clinique et travail de la pensée (De Boeck, Paris, 2008, 41-45)

14. Y. Vacher, Construire une pratique réflexive. Comprendre et agir (De Boeck, Louvain-la-Neuve, 2015)

15. Ph. Blanchet, P. Chardenet (dir), Guide pour la recherche en didactique des langues et des cultures. Approches contextualisées (EAC, Paris, 2011)

16. C. Sheepers, Actes du congrès de l'AREF (2010)

17. D. Bucheton in V. Bigot, L. Cadet, Discours d'enseignants sur leur action en classe. Enjeux théoriques et enjeux de formation (Riveneuve éd., Paris, 2011, 217-224)

18. M. Cambra-Giné, Une approche ethographique de la classe de langue (Crédif-Didier, Paris, 2003)

19. S. Bibauw, J.L. Dufays, Rep.péd., 2, 13 (2010) URL https://halshs.archives-ouvertes.fr/hal00798613/document

20. F. Cicurel, AILE, 16 (2002)

21. L.Cadet, M. Causa in J.C. Beacco, J.L. Chiss, F. Cicurel, D. Véronique (dir.), Les cultures éducatives et linguistiques dans l'enseignement des langues (PUF, Paris, 2005, 159-181)

22. P. Paillé, Le tr. hum., 71 (2008) URL www.cairn.info/revue-le-travail-humain-2008-1-page-22.htm

23. Y. Clot, D. Faïta, Travailler, 4, 11 (2000)

24. F. Gadet, Texto, (2003) URL http://www.revue-texto.net/Inedits/Gadet Principes.html

25. J. Beckerts, N. François in E. Charlier, J. Beckers, S. Boucenna, S. Biemar, N. François, C. Leroy, Comment soutenir la démarche réflexive? (De Boeck, Bruxelles, 2013, 50)

26. F. Cicurel, Le français aujourd'hui, 188, 50 (2015) 